Clinical Medicine

Poster

Abstract ID: 92

\title{
Infected nonunion proximal humerus fracture: two-stage oncologic approach solution with reverse total shoulder reconstruction
}

Khairul Nizam Siron | Goh Kian Liang | Mohd Ariff Sharifuddin | Ed Simor Khan Mor Japar Khan | Mohd Shahidan Nor Rahin | Nik Fatmy Nik Najmy | Aminuddin Che Ahmad | Azril Mohammad Amin

Department of Orthopaedics, Traumatology \& Rehabilitation, Kulliyyah of Medicine, International Islamic University Malaysia

Introduction: Infected non-union of proximal humerus after fixation is devastating consequence and remains challenging. Methods: The staged oncologic approach consists of wide resection and reconstruction ensures remissions of the infection. Results: We report a case of two-stage oncologic approach in a patient with infected non-union proximal humerus after fixation. In the first stage we have performed a wide resection and antibiotic cement spacer insertion. After complete eradication of infection, subsequent reconstruction of the shoulder using reverse shoulder replacement with modular humeral stem had been performed on purpose of restoring the acceptable joint functions. Conclusions: Reconstruction of the infected non-union of the proximal humerus is a challenging task, costly procedure that requires the use of the sophisticated limb reconstruction system. Staged approach incorporating the use oncologic wide resection to eradicate the infection with subsequent bony reconstruction ensure the optimum restoration of upper limb functions.

KEYWORDS: reversed total shoulder replacement, infected non union, proximal humerus 